\title{
Interdisciplinary palliative care for people with advanced Parkinson's disease: a view from the home
}

\author{
Jori E. Fleisher ${ }^{1}$, Ellen C. Klostermann ${ }^{1}$, Serena P. Hess ${ }^{1}$, Jeanette Lee $^{1}$, Erica Myrick ${ }^{1}$, Joshua Chodosh ${ }^{2,3}$ \\ ${ }^{1}$ Department of Neurological Sciences, Rush University Medical Center, Chicago, IL, USA; ${ }^{2}$ Department of Medicine, New York University School \\ of Medicine, New York, NY, USA; ${ }^{3}$ Medicine Service, VA New York Harbor Healthcare System, New York, NY, USA \\ Contributions: (I) Conception and design: JE Fleisher, J Chodosh; (II) Administrative support: EC Klostermann, SP Hess, J Lee, E Myrick; (III) \\ Provision of study materials or patients: JE Fleisher; (IV) Collection and assembly of data: JE Fleisher, EC Klostermann, SP Hess, J Lee, E Myrick; (V) \\ Data analysis and interpretation: JE Fleisher, SP Hess, J Lee, EC Klostermann, J Chodosh; (VI) Manuscript writing: All authors; (VII) Final approval \\ of manuscript: All authors. \\ Correspondence to: Jori Fleisher, MD, MSCE. Department of Neurological Sciences, Section of Movement Disorders, 1725 W. Harrison Street, Suite \\ 755, Chicago, IL 60612, USA. Email: Jori_Fleisher@rush.edu.
}

Background: Advanced Parkinson's disease (PD) is a multidimensional neurodegenerative condition with motor and non-motor symptoms contributing to increasing disability and decreasing quality of life. As the disease progresses, patients may become homebound and estranged from neurological care, with dire consequences. We describe the increasing epidemiologic burden of and individual risks faced by patients with palliative-stage $\mathrm{PD}$ and their caregivers.

Methods: With the aim of mitigating these risks, we designed and iterated two models of interdisciplinary home visits to maintain continuity of care and illuminate the unmet needs and barriers to care faced by this population. We describe both models in detail, with data on feasibility and patient-centered outcomes achieved in the initial model, and baseline characteristics of participants in the ongoing expanded model. Finally, we illustrate the scope and common themes of such palliative care-informed home visits with two cases.

Results: The pilot model involved over 380 visits with 109 individual patients. Among those patients, PD severity worsened by nearly 12 points annually on the Unified Parkinson's Disease Rating Scale (UPDRS), without a corresponding decline in quality of life. In an ongoing study of the second iteration of the model, 52 patient-caregiver dyads have completed their initial visit, with $44 \%$ bearing a diagnosis of dementia and the majority requiring an assistive device or being bedbound. Two cases highlight the critical importance of thorough medication reconciliation and home safety assessment in the comprehensive evaluation and management of such patients.

Conclusions: As our population ages, therapies increase, and the number of individuals living with advanced PD and related disorders grows, so too does the imperative to recognize and address the palliative care needs of such patients and families. For many, home may be a viable, and perhaps optimal, site for this care.

Keywords: Home visits; interdisciplinary; neurodegenerative disease; Parkinson's disease (PD)

Submitted Aug 12, 2019. Accepted for publication Sep 09, 2019.

doi: 10.21037/apm.2019.09.12

View this article at: http://dx.doi.org/10.21037/apm.2019.09.12

\section{Introduction}

The burden of Parkinson's disease (PD) continues to grow: the 2015 Global Burden of Disease Study reported over
6.1 million individuals affected worldwide, representing a 118\% increase since 1990 (1). In the US alone, studies project more than a twofold rise in persons living with PD between 2015 and 2060, to 1.96 million, and a tripling of 
those with $\mathrm{PD}$ and dementia (2). As PD progresses, so, too, do functional impairments, disabling non-motor symptoms, and dementia $(3,4)$.

Recent studies of care partners of people with advanced PD have highlighted the necessity of guidance on disease course, medications, emergencies, and unmet psychosocial needs $(5,6)$. Such patients are at risk of becoming homebound and estranged from expert care, and more prone to adverse events, including emergency room visits and hospitalizations (7-9). Acute care settings often disrupt carefully orchestrated medication schedules and administer contraindicated medications, leading to greater lengths of stay, higher rates of readmission and in-hospital mortality (10-12). Furthermore, this population is at a fivefold increased risk of nursing home placement, with dementia and hallucinations among the greatest predictors (13-15). Once placed in a long-term care facility, only one third of residents with PD continue to receive outpatient neurologist care (14), despite such care being associated with fewer falls and lower mortality (16).

As is highlighted throughout this special issue, applying a palliative care approach to individuals with PD can have significant benefit (17-19). One window of opportunity for intervention is to facilitate continued access to expert care for those who are homebound. In various populations of elderly patients, comprehensive home visit programs for complex chronic conditions have yielded better care quality, patient-centered outcomes, health care utilization, and higher likelihood of dying at home according to patients' wishes (20-24). In light of the above, we designed and adapted an interdisciplinary home visit model for people with advanced PD and their care partners. Here, we describe the first two iterations of this model and provide two case studies to illustrate the challenges and opportunities of this approach.

\section{Methods}

\section{Edmond 7. Safra Interdisciplinary Home Visit Program for Advanced PD}

In 2014, we launched the Edmond J. Safra Interdisciplinary Home Visit Program for Advanced PD (HVP) at the Marlene and Paolo Fresco Institute for Parkinson's and Movement Disorders at New York University to address the gap in continuity of care for homebound individuals with PD. This philanthropically-supported first iteration of the model is described in detail elsewhere (25), and summarized briefly here. Movement disorders specialists referred patients to the program if they were diagnosed with PD or a related disorder, met Medicare homebound criteria (26), lived within New York City, and their neurologist deemed them at high risk of hospitalization or institutionalization.

Patients received quarterly comprehensive visits from a movement disorders specialist, nurse, and social worker. The team identified and addressed motor and non-motor symptoms, medication errors, home safety issues, and unmet psychosocial needs. We implemented a detailed assessment and plan, including referrals to in-home therapies and community resources.

Between February 2014 and July 2017, the HVP team conducted over 380 visits with 109 individual patients. In an analysis of visits through July 2016, the median age of patients at baseline was 79.6 years, $52 \%$ were female, $38 \%$ were rated as Hoehn and Yahr Stage 4 (severe disability, able to ambulate with assistance) and 32\% rated Stage 5 (wheelchair- or bedbound). Medication errors were frequently uncovered through detailed in-home medication reconciliation, with errors of commission-in which the patient was taking a medication that was either recorded as discontinued by the referring provider or which the provider was unaware of-being the most frequent type of error (27). Medication changes were recommended at $42 \%$ of visits, non-pharmacologic changes-such as dietary changes or specific assistive devices-at $45 \%$ of visits, and referrals to therapies or community resources at $93 \%$ of visits. Advance directives were addressed with $90 \%$ of patients. Median satisfaction with the HVP was 96/100 for patients and 98/100 for care partners.

Among a subset of 27 patients followed prospectively for 1 year and who were cognitively intact at baseline, disease severity worsened significantly, as measured by a nearly 12-point increase on the Unified Parkinson's disease Rating Scale (UPDRS) (28). Despite this sharp decline and the typically parallel trajectories of disease severity and quality of life in PD (29), quality of life did not change in this cohort over 1 year (30).

\section{IN-HOME PD}

Despite much success, limitations of model scalability included time- and labor-intensity, one geographic site, and a lack of billing pressure. To address these issues, we launched the second model at Rush University in 2018 with greater geographic diversity-a 30-mile radius encompassing both urban, suburban, and rural areas. 

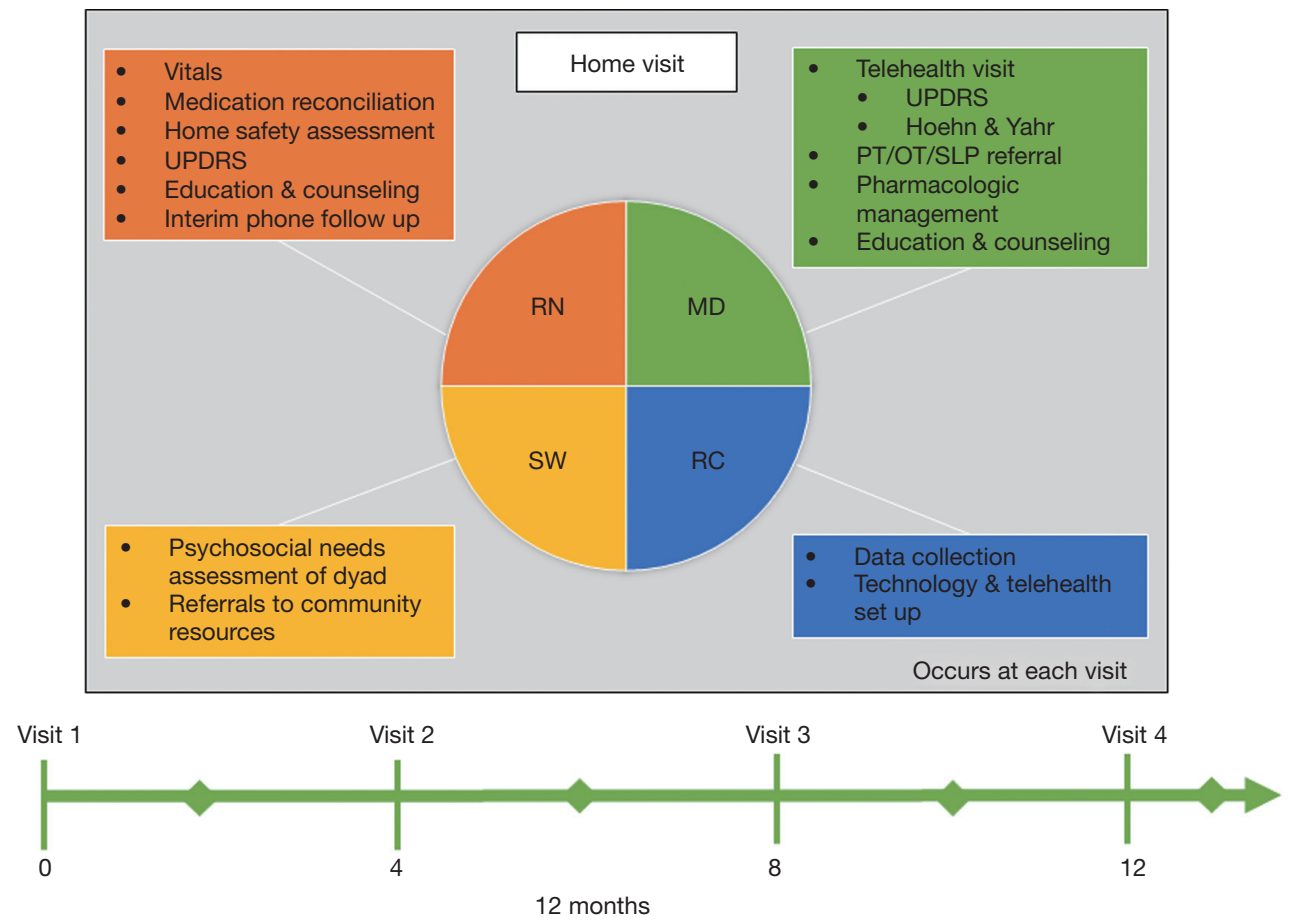

- Phone check-in 2-8 weeks post visit

Figure 1 IN-HOME-PD visit structure, timeline, and discipline-specific responsibilities at each visit. RN, nurse; MD, movement disorders specialist; SW, social worker; RC, research coordinator; UPDRS, Unified Parkinson's Disease Rating Scale; PT, physical therapy; OT, occupational therapy; SLP, speech language pathologist.

While the nurse, study coordinator, and social worker travel in person to the initial visit, the movement disorders specialist is present via telemedicine for all visits, as is the social worker for visits $2-4$, to improve efficiency and costeffectiveness.

In total, 65 patient-caregiver dyads will be enrolled in the Chicago-based model, titled IN-HOME PD (Interdisciplinary Home Visits for Parkinson's disease, K23NS097615, NCT03189459, approved by the Rush University Medical Center Institutional Review Board, \#17080209), depicted in Figure 1. Participants are referred by movement disorders specialists at Rush or identified by chart review, and must meet eligibility criteria (Table 1). Participants enroll for 4 home visits over approximately 1 year and may continue outpatient visits with their referring specialist. However, to avoid duplication of efforts, the home visit team takes over as the primary point of contact. After completing the study enrollment process at the first visit, the team completes questionnaires pertaining to demographics, PD history, comorbidities, healthcare utilization, and quality of life, along with a brief cognitive assessment for each dyad member. The coordinator ensures the readiness of the telemedicine platform, consisting of the Vidyo $^{\circledR}$ video conferencing application installed on a mobile hotspot-connected tablet.

While the coordinator is helping the patient complete questionnaires, the nurse conducts a brief home safety assessment, identifying fall risks and other safety concerns (Figure 2). The nurse completes a comprehensive medication reconciliation, comparing the actual prescriptions, vitamins, supplements, over-the-counter medications, and organization system present in the home with the medications listed in the electronic medical record. The goals are twofold: first, to identify the exact dose, frequency, and timing of all dopaminergic and other PD-related medications to address motor fluctuations, dyskinesias, and adverse effects; second, to identify potentially inappropriate medications (31) and medication errors, as shown in Figure 2, which may be contributing to motor and non-motor symptoms, fall risks, and cognitive decline. Finally, the nurse interviews the dyad using the UPDRS parts 1 and 2 (28). This well-validated scale covers 
Table 1 IN-HOME PD patient and caregiver eligibility criteria

Patient eligibility criteria

At least 40 years old

Primarily English-speaking

Idiopathic Parkinson's disease, Hoehn and Yahr stage 3-5 at last office visit

Community-dwelling within 30-mile radius of Rush University

Homebound per Medicare criteria

Have an unpaid caregiver willing to participate in the study

Have $\geq 1$ of: fluctuations, multi-morbidity, medication mismanagement, cognitive impairment, depression or anxiety, high risk for hospitalization, high risk for nursing facility admission, suspected elder abuse, recent history of increased falls, caregiver burnout

Exclusion criteria

Diagnosis of another neurodegenerative disease

Caregiver eligibility criteria

At least 30 years old

Primarily English-speaking

Unpaid for caregiving role, and cohabitating with or spending an average of $>20$ hours weekly engaged in care-related activities for the patient

Exclusion criteria

Active psychosis or severe psychiatric disorder

Terminal illness (life expectancy $<12$ months)

neuropsychiatric symptoms, speech, swallowing, dressing, hygiene, falling, freezing of gait, and pain, with each item rated 0 (asymptomatic) to 4 (severe).

As the nurse and coordinator are completing their duties, the social worker conducts a detailed psychosocial needs assessment of the dyad, including daily challenges and financial resources and stressors. She details utilization or need for various services, including: caregiver, homemaker, and nutritional services, in-home therapies, medical alert systems, or adult day centers. Finally, the social worker asks about advance directives; if not completed, she provides resources for designating a health care power of attorney and completing a living will or another advance directive.

Once the nurse and social worker have completed their tasks, they call the movement disorders specialist to present the case. After this briefing, the team rejoins the dyad and connects to the neurologist between a Vidyo ${ }^{\circledR}$-enabled tablet and the neurologist's office workstation.

The neurologist reviews the dyad's chief concerns, along with any pertinent positive UPDRS symptoms, medication errors, or safety concerns. She completes the UPDRS motor exam, conducted virtually (32). The neurologist determines the efficacy of the PD medication regimen, including "on" and "off" periods, dyskinesias, and other adverse effects. Goals of care are confirmed before collaborating with the team to propose a comprehensive plan. The team leaves the dyad with a step-by-step written summary, including all medication changes and referrals.

Following the visit, each team member records their findings in the electronic medical record, which is compiled into a comprehensive note shared with all relevant health care providers. The dyad receives contact information to reach the team between visits, and a team member follows up by phone 2-8 weeks post-visit. Visits $2-4$ are identical to Visit 1, except the social worker joins via telemedicine, and the home safety assessment is abbreviated if no changes have occurred.

\section{Results}

To date, 52 dyads have completed Visit 1, with baseline characteristics of patients and caregivers shown in Tables 2,3, respectively. Informed consent was obtained from all patients and caregivers. Notably, $36.5 \%$ of patients are female, with a mean age of 78.6 and disease duration of 15.4 years. Over $44 \%$ of patients carry a diagnosis of PD Dementia at baseline. Overall, 135 visits have been conducted. The mean one-way travel time is 46.8 minutes (SD 29.2).

\section{Case studies}

Each patient in the HVP and IN-HOME PD present unique challenges in terms of complex and bothersome motor and non-motor symptoms, comorbidities, and psychosocial stressors. However, we highlight two cases below illustrating common themes and opportunities for enhanced quality of life uniquely afforded by home visits.

\section{Case study \# 1}

Mr. C is an 82-year old gentleman referred for home visits with a diagnosis of PD for 13 years. He had a history of myelodysplastic syndrome with thrombocytopenia and coronary artery disease status post coronary bypass graft. His PD had been complicated by severe freezing of 


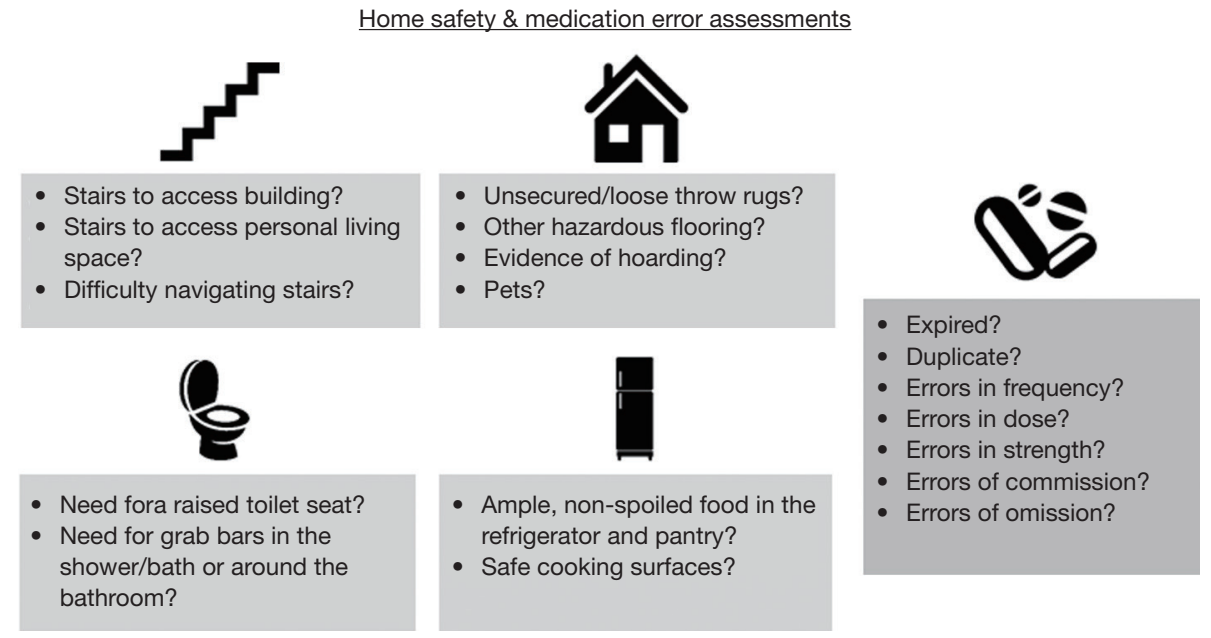

Figure 2 Home safety assessment and medication reconciliation items addressed during home visits.

Table 2 Patient characteristics at baseline visit

\begin{tabular}{lc}
\hline Patient demographics & $\mathrm{N}=52$ \\
\hline Mean age (SD) & $78.6(7.7)$ \\
Female, \% & 36.5 \\
Race, n (\%) & \\
Caucasian & $35(67.3)$ \\
African American/Black & $7(13.5)$ \\
Asian & $7(13.5)$ \\
Hispanic, Caucasian or declined to identify race, $\mathrm{n}(\%)$ & $3(5.8)$ \\
Hoehn and Yahr stage, mean (SD) & $4.0(0.6)$ \\
Disease duration in years, mean (SD) & $15.4(6.2)$ \\
Number of comorbidities, mean (SD) & $4.6(2.0)$ \\
\hline
\end{tabular}

gait, constipation, and prior hallucinations. He lived in a single-family, split-level home with his wife, who served as his primary caregiver, with additional help from their children living nearby. Mr. C's pertinent medications at visit 1 included carbidopa-levodopa, mirtazapine, selegiline, rivastigmine, atorvastatin, and metoprolol tartrate. Medications were organized well into weekly, 4 compartment/day pillboxes. The home was equipped with many safety measures except a shower chair and bathroom grab bars. Mr. C had not received any physical, speech, or occupational therapy within the past year.

Among his most bothersome complaints, Mr. C felt he could only exert himself minimally during the day due to
Table 3 Caregiver demographics

\begin{tabular}{lc}
\hline Caregiver demographics & $\mathrm{N}=52$ \\
\hline Mean age at Visit 1 (SD)* & $66.5(11.3)$ \\
Female, \% & 76.9 \\
Race, $\mathrm{n}(\%)$ & $36(69.2)$ \\
Caucasian & $7(13.5)$ \\
African American/Black & $6(11.5)$ \\
Asian & $3(5.8)$ \\
Hispanic, Caucasian or declined to identify race, $\mathrm{n}(\%)$ & \\
Relationship to patient, $\mathrm{n}$ (\%) & $32(61.5)$ \\
Spouse/partner & $16(30.8)$ \\
Adult child & $1(1.9)$ \\
Other family member & $3(5.8)$ \\
Family friend/neighbor & $2.9(1.9)$ \\
\hline Mean number of comorbidities at Visit 1 (SD)
\end{tabular}

${ }^{*}, \mathrm{n}=49,3$ caregivers declined to answer this question.

continually feeling "light-headed and weak" upon standing. He had 3-5 falls weekly, sometimes several in a day, with extreme bruising. He also endorsed unintentional weight loss of approximately 17 pounds in the preceding 6 months, dropping to 142 pounds. His examination was remarkable for blood pressure 111/65 and heart rate 58 while seated; 99/52, heart rate 61 after standing for 2 minutes. He had numerous scattered ecchymoses on his extremities and $2+$ pitting edema to the ankles bilaterally. He had moderate 
hypophonia, rigidity, and bradykinesia. He was able to push himself up to a standing position but experienced severe freezing of gait despite using a rolling walker.

Our team's assessment confirmed the aforementioned conditions, plus orthostatic hypotension $(\mathrm{OH})$, most of which were contributing to his frequent falls. These were made more dangerous in light of his myelodysplastic syndrome and thrombocytopenia. The team reviewed goals of care, which included aging in place and avoiding hospitalization and institutionalization, with health care proxy, power of attorney, and a do not resuscitate order all active. Given his age, symptoms, prognosis, and goals of care, the team and dyad agreed upon the following plan: for $\mathrm{OH}$ and pedal edema contributing to falls, we weaned his metoprolol, recommended elevating his legs and wearing compression stockings, and referred him to in-home physical therapy with a therapist trained in PD-specific modalities (33). For cognition, we initiated memantine $(34,35)$. We discontinued his statin in an effort to minimize unnecessary polypharmacy (36), and provided nutritional strategies to increase high-density caloric intake to mitigate further weight loss and deconditioning.

By visit 2, his wife stated "his falls have decreased by $80 \%$ and his energy improved" and the patient told his wife "I can tell I am walking better". By elevating his legs and wearing compression stockings, his edema was "almost gone" and he had more interest and ability to move beyond one floor in his home. Mr. C was able to attend and participate in his grandchild's wedding, which he had not thought possible.

\section{Case study \#2}

Mrs. S is a 90-year-old female with a diagnosis of PD for 8 years, complicated by symptomatic $\mathrm{OH}$, prior visual hallucinations, motor fluctuations, and dyskinesias. At Visit 1 , she reported episodic dyspnea for which she had recently been hospitalized and discharged home on continuous oxygen without a diagnosis or plan for follow-up. She endorsed significant unintentional weight loss-as low as 88 pounds during the hospitalization-and constipation, occasional hallucinations, insomnia, and dysphagia which had improved on a mechanical soft diet with thickened liquids. She lived in a one-floor condominium, had a paid caregiver in the morning to early afternoon, but was alone for 2-3 hours before one of her children would come sleep at her home each night. The largest safety concern was that the patient was tethered to a large oxygen compressor, located in her bedroom, with tubing running throughout the entirety of the apartment, creating a significant fall risk. Her pertinent medications included carbidopa-levodopa, amantadine, trazodone, and lisinopril.

Her seated blood pressure was $138 / 73$, heart rate of 89 , dropping to $96 / 60$ with heart rate of 96 upon standing. Notably, she required a pediatric-sized cuff and manual reading due to her small frame and continuous dyskinesias leading to falsely elevated readings with an adultsized automatic cuff. She had bipedal edema, moderate bradykinesia, and was unable to rise without assistance to reposition her oxygen tubing. She had continuous, nonbothersome dyskinesias without subjective dyspnea while seated, only complaining of dyspnea while ambulating with a rolling walker.

Our team recommended stopping lisinopril given her symptomatic $\mathrm{OH}$ and risk-benefit ratio of hypo- rather than hypertension given her age and prognosis. We recommended that she see a pulmonologist regarding the underlying cause of her dyspnea and alternatives to her home oxygen arrangement. Finally, we provided her with counseling on increasing her nutritional intake.

Within a few weeks of discontinuing lisinopril, Mrs. S reported more energy and her in-home physical therapists documented consistent normotension. At visit 2, weight remained stable and the team discontinued amantadine due to lack of efficacy and probable contribution to intermittent hallucinations and bothersome pedal edema. Our team coordinated a referral to a pulmonologist who discontinued continuous oxygen based on same-day pulmonary function test results and recommended nebulizer treatments as needed for dyspnea. This change alone greatly increased Mrs. S' quality of life because she "hated being tethered to that machine". In the absence of the oxygen compressor and its associated cords and tubes, and with resolution of her hallucinations and bipedal edema after stopping amantadine, her fall frequency decreased to zero in the subsequent 8 months.

\section{Discussion}

The IN-HOME PD study is ongoing and will be completed in late 2020. While participants have presented their own unique constellation of symptoms, psychosocial challenges, and goals of care, several common themes have emerged. Many patients experience frequent unexplained falls, which remain one of the greatest risk factors for hospitalization in PD (37). Assessing this concern in the home can yield significant and actionable insight that may be impossible in 
a typical outpatient setting. Obstacles in the patient's home environment can present unappreciated fall risks, including medical equipment, pets, uneven flooring, untethered cords and cables, and staircases and entryways without handrails.

Beyond motor symptoms leading to falls, the prevalence and importance of $\mathrm{OH}$ in this population cannot be underestimated $(38,39)$. While many home visit patients carried a diagnosis of $\mathrm{OH}$ prior to program referral, far more have been undiagnosed and referred to home visits while on mono- or poly-antihypertensive regimens. The first step for clinicians is to recognize and appreciate the variable presentations of $\mathrm{OH}$ (40). Secondly, individuals with advanced PD have frequently lost significant amounts of weight and may have prominent tremor or dyskinesia, both of which can lead to automated and adult-sized cuffs overestimating blood pressure measurements. Manually measuring orthostatic vital signs using a properly fitted cuff can reveal previously undetected $\mathrm{OH}$.

IN-HOME PD patients have a mean of 4.6 comorbidities and significant associated polypharmacy. Greater polypharmacy has been associated with higher symptom burden and worse quality of life (41). Furthermore, polypharmacy may indicate not only unnecessary, but potentially inappropriate medications $(42,43)$. By reconciling prescribed and over-the-counter medications, vitamins, and supplements, home visits permit the identification of previously undetected medication errors and potentially inappropriate medications $(31,44)$. Deprescribing unnecessary or potentially inappropriate medications has been recognized as a positive intervention to manage polypharmacy and improve outcomes, especially in multimorbid older adults (45). This has been wellreceived among our homebound dyads.

Finally, patients and caregivers in these programs have frequently remarked on the loneliness they feel secondary to their homebound status. In addition to the therapeutic recommendations of the home visit team and the in-home allied health professionals to whom we frequently refer, the continuity of care provides respite from social isolation.

\section{Conclusions}

Challenges abound in refining and sustaining a model of care for homebound individuals with PD, including time, travel, and billing. While telemedicine connects many people with PD who are estranged from care by lack of local expertise (46), additional challenges arise when patients have dementia and sensory impairments. Caregivers may be able to mitigate some of these challenges, provided they are not facing similar ones themselves. Future research is necessary to investigate whether partnering with community health workers or home health agencies might improve access and outcomes for this population.

By deploying an interdisciplinary team to the home, we aim to better understand the multidimensional signs and symptoms, caregiver strain, and social isolation contributing to the adverse outcomes in end-stage PD. Our pilot data suggest high satisfaction and stabilization of patient quality of life despite disease progression. Ongoing work, illustrated by the two case studies, highlights the importance of medication reconciliation, home safety assessments, and appropriate monitoring and treatment of $\mathrm{OH}$. As patients and caregivers vary widely in their medical and psychosocial needs throughout the course of $\mathrm{PD}$, so too will various models be necessary to improve life and death with this condition. In the end, however, new insights and strategies for improving care may be found just out of sight and right at home.

\section{Acknowledgments}

This work was made possible by generous funding from donors who recognized the value of extending care beyond the clinic walls, including the Edmond J. Safra Philanthropic Foundation, the Doris Duke Charitable Foundation Fund to Retain Clinical Scientists, National Institute of Neurological Disorders and Stroke, CurePSP, Parkinson Foundation, Parkinson Alliance, and Joyce DeMoose and George Harvey. The authors extend their gratitude to the home visit patients and caregivers across the country for sharing their homes and their trust.

Funding: This work was supported by the Edmond J. Safra Philanthropic Foundation; the Parkinson Foundation; Parkinson Alliance; the Doris Duke Charitable Foundation Fund to Retain Clinical Scientists; and the National Institutes of Health (2L30NS084235-02, 2L30NS084235-03, 2L30NS084235-04; 1K23NS097615). Dr. Fleisher has received research support from the NIH/ NINDS (K23NS097615, L30SN084235, U01NS100610), Feldstein Medical Foundation, Doris Duke Charitable Foundation Fund to Retain Clinical Scientists, CurePSP, private philanthropy, and Biogen. She has received honoraria from the Parkinson Foundation, and has received royalties from UpToDate. Dr. Chodosh has received research support from the National Institutes of Health, New York State Department of Health, and Independence 
at Home, Long Beach, CA. Dr. Chodosh has received honoraria from Gerontological Society of America and he serves on the advisory board of Aging in New York Fund. None of the funding sources had involvement in the collection, analysis, or interpretation of data; in the writing of the report; or in the decision to submit the article for publication.

\section{Footnote}

Conflicts of Interest: The authors have no conflicts of interest to declare.

Ethical Statement: The authors are accountable for all aspects of the work in ensuring that questions related to the accuracy or integrity of any part of the work are appropriately investigated and resolved. The study was approved by New York University Institutional Review Board (\#s16-01339) and Rush University Medical Center Institutional Review Board (17080209). Written informed consent was obtained from all patients.

\section{References}

1. GBD 2015 Neurological Disorders Collaborator Group. Global, regional, and national burden of neurological disorders during 1990-2015: a systematic analysis for the Global Burden of Disease Study 2015. Lancet Neurol 2017;16:877-97.

2. Savica R, Grossardt BR, Rocca WA, et al. Parkinson disease with and without dementia: A prevalence study and future projections. Mov Disord 2018;33:537-43.

3. Hely MA, Morris JG, Reid WG, et al. Sydney Multicenter Study of Parkinson's disease: non-L-dopa-responsive problems dominate at 15 years. Mov Disord 2005;20:190-9.

4. Hely MA, Reid WG, Adena MA, et al. The Sydney multicenter study of Parkinson's disease: the inevitability of dementia at 20 years. Mov Disord 2008;23:837-44.

5. Boersma I, Jones J, Coughlan C, et al. Palliative Care and Parkinson's disease: Caregiver Perspectives. J Palliat Med 2017;20:930-8.

6. Boersma I, Jones J, Carter J, et al. Parkinson disease patients' perspectives on palliative care needs: What are they telling us? Neurol Clin Pract 2016;6:209-19.

7. Hassan A, Wu SS, Schmidt P, et al. High rates and the risk factors for emergency room visits and hospitalization in Parkinson's disease. Parkinsonism Relat Disord 2013;19:949-54.
8. Aminoff MJ, Christine CW, Friedman JH, et al. Management of the hospitalized patient with Parkinson's disease: current state of the field and need for guidelines. Parkinsonism Relat Disord 2011;17:139-45.

9. Chou KL, Zamudio J, Schmidt P, et al. Hospitalization in Parkinson disease: a survey of National Parkinson Foundation Centers. Parkinsonism Relat Disord 2011;17:440-5.

10. Martinez-Ramirez D, Giugni JC, Little CS, et al. Missing dosages and neuroleptic usage may prolong length of stay in hospitalized Parkinson's disease patients. PLoS One 2015;10:e124356.

11. Shahgholi L, De Jesus S, Wu SS, et al. Hospitalization and rehospitalization in Parkinson disease patients: Data from the National Parkinson Foundation Centers of Excellence. PLoS One 2017;12:e0180425.

12. Low V, Ben-Shlomo Y, Coward E, et al. Measuring the burden and mortality of hospitalisation in Parkinson's disease: A cross-sectional analysis of the English Hospital Episodes Statistics database 2009-2013. Parkinsonism Relat Disord 2015;21:449-54.

13. Vossius C, Nilsen OB, Larsen JP. Parkinson's disease and nursing home placement: the economic impact of the need for care. Eur J Neurol 2009;16:194-200.

14. Safarpour D, Thibault DP, DeSanto CL, et al. Nursing home and end-of-life care in Parkinson disease. Neurology 2015;85:413-9.

15. Aarsland D, Larsen JP, Tandberg E, et al. Predictors of nursing home placement in Parkinson's disease: a population-based, prospective study. J Am Geriatr Soc 2000;48:938-42.

16. Willis AW, Schootman M, Tran R, et al. Neurologistassociated reduction in PD-related hospitalizations and health care expenditures. Neurology 2012;79:1774-80.

17. Miyasaki JM, Long J, Mancini D, et al. Palliative care for advanced Parkinson disease: an interdisciplinary clinic and new scale, the ESAS-PD. Parkinsonism Relat Disord 2012;18 Suppl 3:S6-9.

18. Kluger BM, Persenaire MJ, Holden SK, et al. Implementation issues relevant to outpatient neurology palliative care. Ann Palliat Med 2018;7:339-48.

19. Kluger BM, Katz M, Galifianakis N, et al. Does outpatient palliative care improve patient-centered outcomes in Parkinson's disease: Rationale, design, and implementation of a pragmatic comparative effectiveness trial. Contemp Clin Trials 2019;79:28-36.

20. Edes T, Kinosian B, Vuckovic NH, et al. Better access, quality, and cost for clinically complex veterans with home- 
based primary care. J Am Geriatr Soc 2014;62:1954-61.

21. Kono A, Kanaya Y, Tsumura C, et al. Effects of preventive home visits on health care costs for ambulatory frail elders: a randomized controlled trial. Aging Clin Exp Res 2013;25:575-81.

22. Boult C, Green AF, Boult LB, et al. Successful models of comprehensive care for older adults with chronic conditions: evidence for the Institute of Medicine's "retooling for an aging America" report. J Am Geriatr Soc 2009;57:2328-37.

23. DeCherrie LV, Soriano T, Hayashi J. Home-based primary care: a needed primary-care model for vulnerable populations. Mt Sinai J Med 2012;79:425-32.

24. Tanuseputro P, Beach S, Chalifoux M, et al. Associations between physician home visits for the dying and place of death: A population-based retrospective cohort study. PLoS One 2018;13:e0191322.

25. Fleisher J, Barbosa W, Sweeney MM, et al. Interdisciplinary Home Visits for Individuals with Advanced Parkinson's disease and Related Disorders. J Am Geriatr Soc 2018;66:1226-32.

26. Pub 100-02 Medicare Benefit Policy: Clarification of the Confined to the Home Definition in Chapter 15, Covered Medical and Other Health Services, of the Medicare Benefit Policy Manual 2014. Available online: https:// www.cms.gov/Regulations-and-Guidance/Guidance/ Transmittals/downloads/R192BP.pdf

27. Medication errors: The role of the nurse in an interdisciplinary home visit program for advanced Parkinson's disease patients. MDS Abstr. Available online: https://www.mdsabstracts.org/abstract/medication-errorsthe-role-of-the-nurse-in-an-interdisciplinary-home-visitprogram-for-advanced-parkinsons-disease-patients/

28. Fahn S, Elton RJ, Committee UD. The Unified Parkinson's disease Rating Scale. In: Fahn S, Marsden CD, Calne DB, et al., editors. Recent Dev Park Dis Florham Park, NJ: Macmillan Healthcare Information; 1987.

29. Martínez-Martín P, Rodríguez-Blázquez C, Forjaz MJ, et al. Relationship between the MDS-UPDRS domains and the health-related quality of life of Parkinson's disease patients. Eur J Neurol 2014;21:519-24.

30. Fleisher JE, Sweeney MM, Oyler S, et al. Disease Severity And Quality Of Life In Homebound People With Advanced Parkinson's disease: A Pilot Study. Neurol Clin Pract 2019. [Epub ahead of print].

31. By the 2019 American Geriatrics Society Beers Criteria ${ }^{\circledR}$ Update Expert Panel. American Geriatrics Society 2019 Updated AGS Beers Criteria ${ }^{\circledR}$ for Potentially
Inappropriate Medication Use in Older Adults. J Am Geriatr Soc 2019;67:674-94.

32. Abdolahi A, Scoglio N, Killoran A, et al. Potential reliability and validity of a modified version of the Unified Parkinson's disease Rating Scale that could be administered remotely. Parkinsonism Relat Disord 2013;19:218-21.

33. Fox C, Ebersbach G, Ramig L, et al. LSVT LOUD and LSVT BIG: Behavioral Treatment Programs for Speech and Body Movement in Parkinson Disease. Parkinsons Dis 2012;2012:391946.

34. Meng YH, Wang PP, Song YX, et al. Cholinesterase inhibitors and memantine for Parkinson's disease dementia and Lewy body dementia: A meta-analysis. Exp Ther Med 2019;17:1611-24.

35. Larsson V, Engedal K, Aarsland D, et al. Quality of life and the effect of memantine in dementia with lewy bodies and Parkinson's disease dementia. Dement Geriatr Cogn Disord 2011;32:227-34.

36. Kutner JS, Blatchford PJ, Taylor DH, et al. Safety and benefit of discontinuing statin therapy in the setting of advanced, life-limiting illness: a randomized clinical trial. JAMA Intern Med 2015;175:691-700.

37. Koay L, Rose J, Abdelhafiz AH. Factors that lead to hospitalisation in patients with Parkinson disease-A systematic review. Int J Clin Pract 2018;72:e13039.

38. Palma JA, Kaufmann H. Treatment of autonomic dysfunction in Parkinson disease and other synucleinopathies. Mov Disord 2018;33:372-90.

39. Palma JA, Gomez-Esteban JC, Norcliffe-Kaufmann L, et al. Orthostatic hypotension in Parkinson disease: how much you fall or how low you go? Mov Disord 2015;30:639-45.

40. Gibbons CH, Schmidt P, Biaggioni I, et al. The recommendations of a consensus panel for the screening, diagnosis, and treatment of neurogenic orthostatic hypotension and associated supine hypertension. J Neurol 2017;264:1567-82.

41. Schenker Y, Park SY, Jeong K, et al. Associations Between Polypharmacy, Symptom Burden, and Quality of Life in Patients with Advanced, Life-Limiting Illness. J Gen Intern Med 2019;34:559-66.

42. Thorpe JM, Thorpe CT, Kennelty KA, et al. The impact of family caregivers on potentially inappropriate medication use in noninstitutionalized older adults with dementia. Am J Geriatr Pharmacother 2012;10:230-41.

43. Lindsay J, Dooley M, Martin J, et al. Reducing potentially inappropriate medications in palliative cancer patients: evidence to support deprescribing approaches. Support 
Care Cancer 2014;22:1113-9.

44. Anderson K, Stowasser D, Freeman C, et al. Prescriber barriers and enablers to minimising potentially inappropriate medications in adults: a systematic review and thematic synthesis. BMJ Open 2014;4:e006544.

45. Reeve E, Gnjidic D, Long J, et al. A systematic review of

Cite this article as: Fleisher JE, Klostermann EC, Hess SP, Lee J, Myrick E, Chodosh J. Interdisciplinary palliative care for people with advanced Parkinson's disease: a view from the home. Ann Palliat Med 2020;9(Suppl 1):S80-S89. doi: 10.21037/ apm.2019.09.12 the emerging definition of "deprescribing" with network analysis: implications for future research and clinical practice. Br J Clin Pharmacol 2015;80:1254-68.

46. Achey M, Aldred JL, Aljehani N, et al. The past, present, and future of telemedicine for Parkinson's disease. Mov Disord 2014;29:871-83. 